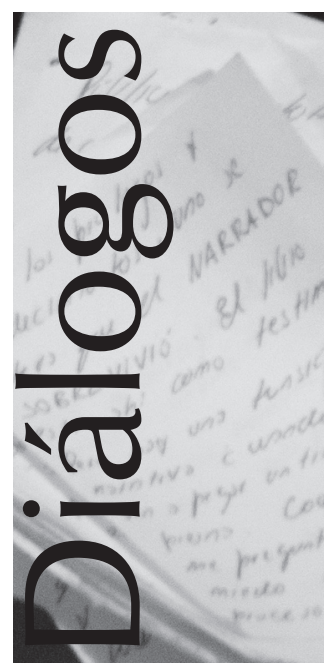

\title{
Práctica editorial contextualizada: Carlos Augusto Monteiro y la Revista de Saúde Pública
}

\author{
Contextualized editorial practices: Carlos \\ Augusto Monteiro and the journal Revista \\ de Saúde Pública
}

Viviana Martinovich ${ }^{7}$

RESUMEN Este texto es parte de una serie de entrevistas cuyo propósito es relevar diversas experiencias editoriales y las dificultades similares por las que atraviesan las revistas latinoamericanas, de manera de comenzar a encontrar soluciones contextualizadas que permitan articular esfuerzos aislados. En esta entrevista realizada en julio de 2015, en el Instituto de Salud Colectiva de la Universidad Nacional de Lanús, Carlos Augusto Monteiro nos habla del modelo de financiamiento, el proceso de trabajo, las innovaciones técnicas, la conformación de equipos y sus roles. Asimismo, analiza la relevancia de las revistas latinoamericanas para la difusión de investigaciones de interés de la agenda nacional, y el vínculo entre el desempeño de las revistas, la calidad de los posgrados y las investigaciones.

PALABRAS CLAVES Revistas Electrónicas; Políticas Editoriales; Edición; Sistemas de Créditos y Evaluación de Investigadores; Sistemas de Evaluación de las Publicaciones; Brasil.

ABSTRACT This text is part of a series of interviews that seek to explore diverse editing and publication experiences and the similar difficulties Latin America journals face, in order to begin to encounter contextualized solutions that articulate previously isolated efforts. In this interview, carried out in July 2015 in the Instituto de Salud Colectiva [Institute of Collective Health] of the Universidad Nacional de Lanús, Carlos Augusto Monteiro speaks to us about funding, work processes, technological innovations, and establishing teams and roles. He analyzes the importance of Latin American journals as a platform for spreading research relevant to national agendas, and the connection between journal performance, the quality of graduate training programs and research quality.

KEY WORDS Electronic Journals; Editorial Policies; Publishing; Researcher Performance Evaluation Systems; Systems for Evaluation of Publications; Brazil. 


\section{INTRODUCCIÓN}

Carlos Augusto Monteiro es médico y magíster en Medicina Preventiva. Realizó el doctorado en Salud Pública en la Universidade de São Paulo (USP), y el posdoctorado en el Institute of Human Nutrition, de Columbia University. Es profesor titular del Departamento de Nutrição de la Faculdade de Saúde Pública de la USP y, desde 1992, coordinador científico del Núcleo de Pesquisas Epidemiológicas em Nutrição e Saúde. Dentro de su producción científica se destacan estudios sobre condiciones de salud y nutrición de la población brasileña, patrones de alimentación y salud en Brasil, y transición alimentaria y nutricional en países en desarrollo. Desde hace más de diez años, es editor científico de la Revista de Saúde Pública, una de las revistas científicas brasileñas más relevantes del campo de la salud pública.

En julio de 2015, Monteiro fue convocado por el Instituto de Salud Colectiva (ISCo) de la Universidad Nacional de Lanús para dictar un seminario en el marco del proyecto "Abordajes multinivel y de curso de vida para la prevención de enfermedades no transmisibles (2012-2017)" realizado en colaboración con University of Michigan, Drexel University y el Instituto de Nutrición de Centro América y Panamá (INCAP) de Guatemala, financiado por Fogarty International Center y National Institute on Aging, dependientes de los National Institutes of Health (NIH) de EE.UU.

Al finalizar una de las jornadas del seminario, nos reunimos en el ISCo para dialogar sobre el surgimiento de la Revista de Saúde Pública, el modo de producción, la política editorial, y la incidencia de las políticas de ciencia y tecnología brasileña en la producción editorial.

\section{DIÁLOGO}

\section{Surgimiento de la Revista de Saúde Pública}

Viviana Martinovich: La Revista de Saúde Pública surge a fines de la década de 1960...
Carlos Augusto Monteiro: La revista cumple 50 años en 2016 y esto explica en parte su éxito. No es fácil que algo dure 50 años en Brasil y la revista viene mejorando cada vez más. Al analizar los indicadores de desempeño se visualiza un proceso de crecimiento, y por detrás de eso hay personas.

El fundador de la revista fue el profesor Oswaldo Forattini, médico entomólogo, un profesional muy competente en su área y una persona visionaria en el sentido de decir "no quiero hacer una cosa más o menos, tiene que ser una de las mejores revistas; puede demorar un tiempo para llegar a serlo, pero ese es el objetivo". Él ya tenía esa impronta y era muy trabajador. Su compañera en esta misión era Maria Teresinha de Andrade, directora de la biblioteca y profesora de la Faculdade de Saúde Pública, quien continúa siendo hasta hoy la directora ejecutiva de la revista. Él fue el editor científico durante cuatro décadas, hasta el año 2000, cuando falleció. Yo tuve el honor y la gran responsabilidad de sustituirlo. Forattini y Teresinha compartían la misma idea de hacer siempre mejor las cosas. Esa búsqueda de la excelencia es una idea vinculada al propio proyecto de la USP, que se origina en los años treinta en un grupo de intelectuales de San Pablo, sobre todo del área de filosofía, que tenía mucha influencia francesa, y que incluso trajeron profesores de Francia, por lo que la USP siempre fue una universidad de excelencia, y la revista se inscribe en esa línea.

De allí que siempre hubo una preocupación muy grande por el cumplimiento de la periodicidad, por la puntualidad en la publicación, por sostener un estándar, un patrón de calidad, siempre fue así. Por ejemplo, hoy todos los artículos se publican en inglés, las personas pueden presentar los artículos en español, portugués o inglés, y en aquellos casos en que no estén en inglés, se traducen y se publican en forma electrónica en dos idiomas. De hecho, ahora solo tenemos la versión electrónica, ya no tenemos versión en papel.

La decisión de abandonar la versión impresa tuvo ciertos desacuerdos, pero la cuestión es el financiamiento de la revista. ¿Cómo se financia una revista? Cada vez es 
más interesante la cuestión tecnológica, pero no abarata el costo sino que, por el contrario, lo aumenta, porque complejiza el proceso. La realidad es que lo único que logramos economizar fue el papel.

Todos esos avances que fueron surgiendo y que pasaron a ser accesibles, la revista fue la primera probablemente en adoptarlos y hacer el esfuerzo necesario para acompañar cada nuevo proceso. Por ejemplo, respecto de la traducción, creo que es la única revista del área que tiene todos los artículos traducidos al inglés, y claro que eso aumenta la visibilidad del artículo, es obvio, el idioma de la ciencia hoy es el inglés.

\section{Modo de producción}

VM: A partir de 2012 cambian el modelo de financiamiento y comienzan a cobrar a los autores. ¿Cómo fue ese pasaje?

CAM: Ese fue un paso muy difícil de dar, relacionado al funcionamiento de la revista. Es una revista de la USP y la universidad nos aporta trabajadores, en nuestro caso contamos con una sola empleada y el resto es trabajo voluntario. Los editores asociados, editores científicos, editores ejecutivos, nadie gana nada. Algunos de ellos son profesores de la facultad, muchos otros no, y todos hacen trabajo voluntario. Pero para producir la revista, además del costo de diagramación -que en nuestro caso se terceriza a través de una empresa- el trabajo más caro es el de corrección de estilo, que se realiza luego de que el artículo fue aprobado. Los autores no siempre escriben de modo claro.

La corrección de estilo es un paso posterior al trabajo realizado por los revisores científicos, quienes se ocupan principalmente de cuestiones más técnicas. Es importante decir que, si bien a veces el proceso de revisión puede prolongarse mucho, nunca se aprueba un artículo sin reunir los pareceres que sean necesarios. Sin embargo, tanto el editor asociado como los revisores no siempre tienen en cuenta las cuestiones idiomáticas, la claridad expositiva, los estándares de presentación de tablas, etc., por lo que contamos con dos personas que trabajan en esto, y ahora el trabajo se duplicó porque hay que hacerlo tanto en portugués como en inglés.

Cuando comenzamos a traducir los artículos al inglés, el costo de producción de la revista creció mucho. En un comienzo, era el autor quien pagaba la traducción, si decidía traducirlo. Pero ese modelo no garantizaba que todos los contenidos estuvieran en inglés, por lo que cambiamos el modelo, y lo que se les cobra a los autores ahora alcanza casi exclusivamente para traducir el texto al inglés.

VM: En la página web de la revista mencionan que el monto que se cobra se destina fundamentalmente a tres aspectos: al mantenimiento del sistema electrónico de presentación y evaluación de artículos, a la corrección de la redacción científica y a la traducción al inglés. Sin embargo, parecería alcanzar solo para la traducción al inglés.

CAM: La realidad es que el costo de la revista es muy alto. Cada número cuesta más o menos 100.000 reales (unos 30.000 dólares). Es mucho dinero.

VM: Y esos valores, sin papel...

CAM: Dejar de publicar la versión impresa redujo muy poco el costo, se redujo algo, pero muy poco. Pero ¿cómo conseguimos financiamiento?, ¿de dónde provienen los recursos? La USP, además de aportar una empleada, tiene un programa de apoyo a todas las revistas científicas de la universidad, y en estos últimos años crearon un ranking de revistas científicas que las pondera en cinco grupos y nosotros estamos en el primer grupo, por lo que recibimos un valor proporcional.

Otra fuente de ingreso es el Ministerio de Salud, sin este dinero no conseguiríamos editar la revista. En este caso, se firman convenios anuales, cada año tenemos que negociar un nuevo convenio, y ellos tienen dificultades también porque Brasil tiene una gran cantidad de revistas. 
VM: Para el trabajo de corrección y edición de los artículos ¿tienen una sola empleada financiada por la USP y tercerizan el resto de los procesos?

CAM: Siempre es un sacrificio juntar el dinero, un poco de aquí, otro poco de allá. La Fundação de Amparo à Pesquisa do Estado de São Paulo (FAPESP) es otra entidad que aporta fondos, pero solo en el caso de artículos cuya autoría corresponda a personas del estado de São Paulo. Y así se va juntando. Nosotros teníamos personas contratadas de forma precaria que trabajaban unos meses y se iban, y como se trata de una tarea que requiere de gente especializada que sepa hacer el trabajo, la profesora Teresinha las formaba y al poco tiempo se iban y de nuevo había que formar a otras personas. Entonces, para evitar eso, se formó a dos personas y las contratamos formalmente. Ese proceso comenzó con la Secretaría de Salud de São Paulo, cuando conseguimos un convenio por cinco años, por el cual la Secretaría pagaba el sueldo de esas dos personas. Pero al finalizar el período, el nuevo secretario no quiso renovar el convenio y ¿cómo le íbamos a pagar a las dos empleadas? Con el dinero del Ministerio no se puede pagar personal, está prohibido. $\mathrm{Y}$ ahí fue cuando decidimos cobrarle al autor. Cobramos 1.500 reales, que son unos 500 dólares. Si la persona no puede pagar, no se queda sin publicar. De todos modos, los valores no son tan altos, si son cinco autores ponen 100 dólares cada uno.

VM: En Brasil, dentro de los fondos de investigación cuentan con partidas para pagar la publicación...

CAM: Exacto, pero también hay casos en los que los autores no cuentan con fondos como, por ejemplo, los estudiantes que están sin beca $y$, en esos casos, se los exime del pago. El dinero que recibimos de los autores entra como "ingresos industriales" a través de una fundación de apoyo a la Faculdade de Saúde Pública, entonces el dinero ingresa y es la fundación la que contrata a esas dos personas.
VM: En la USP se publican más 130 títulos de revistas. ¿Tienen algún sistema de producción editorial centralizado o cada revista organiza su propio proceso editorial?

CAM: Ellos querían hacer un trabajo centralizado y tuve algunas reuniones con la gente del Sistema Integrado de Bibliotecas de la USP que coordina esa área. Sería muy interesante, no sé si concentrar todo, pero en el caso de las traducciones, podrían contratar una empresa de buena calidad y hacer un paquete con todas las revistas que quieren traducir sus contenidos y negociar: "vamos a tener 500 artículos por año y queremos un mejor precio". Entonces, en vez de pasarnos el dinero y que nosotros individualmente contratemos a las empresas, que es complicadísimo, podrían hacer una licitación internacional, incluso para otras cosas, como el sistema de gestión electrónica de artículos.

VM: ¿Ustedes utilizan algún sistema electrónico de gestión?

CAM: Cuando comenzaron a surgir estas plataformas de gestión, quisimos incorporar esa innovación y en aquel momento desarroIlamos nuestro propio sistema, solo para nosotros, lo cual es un problema porque cada vez que uno quiere hacer un cambio o mejorar el sistema es muy costoso, por lo que ahora vamos a migrar a ScholarOne. La idea es migrar de a poco, comenzar con algunos artículos para ver cómo funciona y, paulatinamente, ir desactivando el sistema anterior.

VM: La revista está disponible en PubMed Central (PMC), lo que requiere de un procesamiento técnico especial de los artículos. ¿Ese trabajo lo tercerizan a través de alguna empresa?

CAM: Esa fue una de las últimas innovaciones que hicimos, que aumentó un poco más el dinero que gastamos [risas...] pero lo conseguimos. Peleamos para poder ingresar a PubMed Central porque son muy rigurosos. Mandamos un número procesado, lo evaluaron y lo aprobaron. Eso lo hace la 
profesora Teresinha. Cuando aparece algo nuevo ella dice: "lo tenemos que hacer" y después vemos de dónde sacamos el dinero [risas...].

VM: ¿Cuál es la función del equipo de editores asociados en el proceso de revisión?

CAM: Cuando comenzamos a recibir muchos artículos, llegaban y se reenviaban a los editores asociados. En ese esquema, el editor científico era un editor asociado más, al que se le asignaba el seguimiento de una parte de los artículos. Pero llegó un momento en que no dimos más abasto. Publicamos seis números por año, con unos 20 a 25 artículos por número, lo que da unos 130 artículos, y rechazamos más o menos entre un $80 \%$ y un $85 \%$, por lo que recibimos casi 1.000 artículos por año. Es mucho, por eso creamos lo que denominamos "preanálisis". Entre los editores científicos -en un comienzo estaba solo yo, después con el tiempo se incorporó la doctora Rita Barradas, y ahora somos tres- nos repartimos esos 1.000 artículos de acuerdo con el área de cada uno y miramos el resumen y el tema, y lo que primero detectamos es que muchas cosas no son del área de salud pública. Como la revista tiene impacto, las personas del área de clínica o de otras áreas envían artículos, y es una pena pero tenemos que decirles: "su artículo no es de salud pública". Contamos con ciertos criterios, por ejemplo, con respecto a la validación de instrumentos que es una cosa que se utiliza mucho en las tesis, que es algo propio de los posgrados y que generó una cantidad de contribuciones. Si es un instrumento de evaluación en salud pública, entonces lo consideramos, pero si es un instrumento para medir calidad de vida del diabético, es un instrumento clínico, no de salud pública, por lo que debería publicarse en una revista de diabetes. Entonces, en el preanálisis se rechazan todos los artículos que no sean de salud pública y todos los artículos que no sean lo suficientemente innovadores. Por ejemplo, un estudio sobre obesidad en dos escuelas de San Pablo sería un trabajo aceptado en una maestría, pero no tiene mucho sentido seguir publicando trabajos sobre el mismo tema. Entonces, tomamos en cuenta las conclusiones, porque si la conclusión dice que la desnutrición es más común en familias pobres, es decir, si son constataciones de la realidad...

Si la pregunta es interesante pasa a revisión, si la pregunta no es tan interesante pero los resultados son interesantes también pasa, pero el resto queda afuera. Entonces, el rol del editor científico actualmente en la revista es ese: reenviar a los editores asociados los artículos que pasaron esa primera etapa de preanálisis. En este momento contamos con unos 15 o 20 editores asociados de áreas bien específicas ¿y qué es lo que hacen?

Lo primero que puede hacer un editor asociado es rechazar el artículo, puede decir: "conozco el área y esto no va". Es raro, pero puede suceder. Si en cambio considera que el artículo puede ir, lo lee, puede decir que metodológicamente es débil y se rechaza o, lo que es más común, lo envía al menos a dos revisores y, al conocer el área, identifica mejor a las personas. Ahí surge el gran problema de conseguir buenas revisiones, un problema que ustedes deben conocer también. Para conseguir dos revisiones es necesario contactar a diez revisores, es un tema al cual no le vemos solución, un tema muy difícil que muchas veces obliga al editor a hablar personalmente con los revisores para decirles: "te mandé un artículo ¿no podrías mandar tus comentarios?". Conseguimos una mejor respuesta con gente más joven, con doctores recién formados. Una vez que el editor asociado consigue las dos revisiones, las evalúa y decide si excluye alguna revisión porque es muy mala y utiliza solo una, o saca algún fragmento que no tenga mucho sentido, y comienza a editar la evaluación que el autor va a recibir. Luego, le envía los comentarios al editor científico que le derivó ese artículo, para ver si concuerda con el resultado, antes de ser enviado a los autores y, en general, las opiniones coinciden.

Nosotros creamos estas cosas en función de la realidad, de la necesidad. Ese cuerpo de editores asociados se renueva cada cuatro años, y la última renovación fue interesante 
porque invitamos a 20 personas que son referentes en cada área, son los mejores de Brasil, y nadie nos dijo que no, ninguno dijo: "estoy ocupado, no puedo". Pero si están ellos, ¿por qué incorporamos el preanálisis? Para que no les lleguen artículos que ya sabemos que no se van a publicar. Entonces, de esta manera, reciben artículos que son de su área, de buena calidad y es más factible que consigan las revisiones. Hay que ir ajustando los procesos a la realidad, y la realidad es que la revista aumentó mucho el número de artículos recibidos, pero ese aumento no es equiparable a un aumento en la calidad de los artículos.

\section{Política editorial y política de ciencia y tecnología}

VM: Ustedes reciben un alto porcentaje de artículos de Brasil...

CAM: La mayoría de los artículos son de Brasil. También recibimos artículos de América Latina, algunos de México, de Argentina, de Colombia y, con cierta frecuencia, de Portugal y de países africanos. Pero el gran porcentaje es de Brasil y ahí surge una gran discusión por las diferencias de criterios con respecto a SciELO. Nosotros también estamos preocupados por la internacionalización de la ciencia, pero lo vemos desde una perspectiva diferente: no tenemos ninguna expectativa de que la Revista de Saúde Pública vaya a publicar un alto porcentaje de artículos extranjeros, ni de autores de América Latina. Va a publicar la producción brasileña, y la de países latinoamericanos, sobre todo de aquellos que no tengan sus propias revistas. Autores estadounidenses, ingleses, australianos ¿por qué van a querer publicar en Revista de Saúde Pública? Es diferente de otras áreas.

Nosotros queremos artículos de buena calidad, queremos publicar las mejores investigaciones en salud pública que se realizan dentro de Brasil. Queremos también publicar buenos artículos que resulten de colaboraciones entre científicos brasileños y científicos de otros países, pero es probable que esos artículos no superen el $15 \%$ o el $20 \%$, y no estamos preocupados por aumentar ese porcentaje. Pero la presión de SciELO es muy fuerte, ellos quieren un $50 \%$ de artículos del exterior. ¿Por qué un estadounidense que trabaja en una universidad estadounidense y tiene diez revistas de calidad en su país va a mandar un artículo a una revista brasileña? Y ahí creo que es diferente el área de salud pública respecto de otras áreas. En Brasil, hay revistas de física, de matemática, de química e incluso de medicina en las que ese planteo puede llegar a tener sentido. Puede ser que haya una revista de física tan buena que un investigador de Inglaterra quiera publicar en ella, porque la física es igual en todo el mundo.

VM: ¿No es una cuestión también de agendas de investigación?

CAM: Justamente es eso. Hago una investigación sobre el impacto del consumo del crack en San Pablo que, para la salud pública brasileña, es fundamental $y$, en términos de relevancia, obtiene 10 puntos aquí. Pero si mando esa misma investigación a una revista inglesa, ellos dicen: "bueno, es interesante, pero cuál es la diferencia con el artículo de Medellín o de Buenos Aires. Ya publiqué la semana pasada uno similar". Entonces, la relevancia en este caso es diferente a la de áreas como la física y la química en las que no es tan clara la diferenciación. En el caso de la salud pública hay artículos que van a ser considerados relevantes en Brasil y no necesariamente van a ser considerados relevantes fuera del país. Por eso es que siempre vamos a publicar más artículos brasileños.

VM: Las revistas editadas en Latinoamérica serían relevantes para cada país porque permitirían revalorizar una agenda propia de investigación...

CAM: Tal cual, porque también se podría decir: "¿para qué tener revistas?". Mi respuesta al respecto es que es necesario tener revistas justamente por eso, porque queremos 
tener en salud pública gente haciendo investigaciones relevantes para la salud pública brasileña. Y los investigadores hacen eso, tienen convocatorias y financiamiento, identifican las prioridades, hacen la investigación, mandan los resultados a una revista extranjera de esas de primera línea, y ¿qué sucede?: el trabajo puede no ser considerado muy relevante porque ellos están preocupados por cuestiones más globales y no aceptan ese artículo. Si no hubiera revistas de calidad en Brasil, ese autor tendría que mandar su artículo a revistas que no tienen buenos procesos de revisión y corrección. Y esa es otra cuestión importante: el proceso de revisión mejora la calidad de la investigación, los comentarios y las modificaciones forman parte del proceso de perfeccionamiento, y solo así funciona realmente.

Todos los países, sin excepción, en el área de salud pública, necesitan tener revistas de buena calidad editadas en su propio país que ayuden a aumentar la calidad del material que se difunde, y que estimulen a las personas a hacer investigaciones de interés para la agenda nacional. Porque si el país no tiene revistas el autor va a decir: "voy a orientar mi investigación a lo que le interesa a The Lancet, ellos están publicando artículos en la temática de nutrigenética, entonces voy a trabajar sobre nutrigenética". Por eso es que cada país necesita tener sus propias revistas.

$Y$ ahí surge otro tema: para poder tener revistas, hay que tener recursos. ¿Y vale la pena tener cualquier tipo de revista? Creo que no. Hay personas que dicen: "es bueno tenerlas, porque si no puede publicar en esta, publica en la otra". Pero esa otra no puede ser tan mala, porque si esa revista publica el artículo tal como se presentó, produce el efecto contrario y el autor considera que está haciendo las cosas tan bien que no necesita mejorar nada: "tengo 30 artículos ya publicados, voy viento en popa" [risas...]. Creo que no se puede hacer eso, creo que la revista debe exigirle al autor.

Lo mismo sucede con la relevancia, lo cual genera un tema complicado. Los posgrados se expandieron mucho en Brasil, y se crearon cursos en todas las universidades, y algunas universidades deberían tener carreras de grado pero no de posgrado porque no tienen condiciones para tener posgrados. Tal vez en un futuro tengan condiciones, pero por ahora no y entonces no quieren abrir un posgrado. Pero si no tienen un posgrado, no entran en el sistema nacional de evaluación de investigadores $y$, entonces, abren posgrados de manera precoz y comienzan a tener alumnos que aceptan ir a esa escuela que aún no tiene experiencia para construir una maestría. Hacen la maestría y tienen que publicar, porque si no la Coordenação de Aperfeiçoamento de Pessoal de Nível Superior (CAPES) no evalúa el programa, y esos artículos los envían a nuestra revista. ¿Y qué es lo que ellos mandan? Lo que hizo el estudiante. ¿Y qué hizo el estudiante? Lo que es posible hacer dentro de las condiciones en las que estaba. Y hay personas que dicen: ¿y dónde va a publicar? Él hizo una maestría, la función de las revistas es publicar esa producción de los posgrados, pero si la publicación de los posgrados son ejercicios... En los posgrados hay que enseñar a investigar y lo primero que hay que enseñar es qué es investigación y qué no es investigación. La investigación no puede hacerse sobre cualquier cosa, no es cualquier resultado, si no tiene una pregunta relevante no hay investigación. Entonces, en muchos programas de posgrado, la investigación es un ritual, el docente dice: "está este instrumento, está ese grupo ahí, andá y aplicalo". Ahí el estudiante lo aplica, hace las tablas y dice "hallé tal cosa, igual que lo que todos están encontrando...". Pero él no tenía una pregunta, sus resultados van a tener cero citaciones. ¿Por qué alguien va a citar eso? Y lo que es peor es que se le está diciendo que eso es investigar y lo que es probable es que él les enseñe a otros estudiantes lo mismo. Entonces, creo que todos los programas de posgrado tienen que enseñar a investigar y eso significa tener una pregunta relevante y producir alguna cosa relevante, no puede ser solo un ejercicio. $Y$ nosotros pagamos ese precio en la revista, porque una parte muy grande de los artículos que se presentan no son de investigación, no tienen una pregunta. 
VM: La Revista de Saúde Pública tiene un factor de impacto de 1,219 y con los nuevos criterios Qualis-Capes fue categorizada como Qualis A2 en el área de Saúde Coletiva. ¿Cómo ven desde Brasil que los criterios de evaluación elaborados por el propio país provoquen que sus mejores revistas no sean categorizadas en la categoría A1?

CAM: No veo ningún problema en eso, creo que hay que estimular a los investigadores a que intenten publicar alguno de sus artículos en las revistas consideradas $\mathrm{A} 1$, porque es parte de la formación científica de ellos. Es necesario tener grupos de excelencia y en Brasil los tenemos. Cuando se produjo ese descenso de las revistas brasileñas no me preocupé porque sé lo que es publicar un artículo en una revista como Lancet y lo que es publicar un artículo en la Revista de Saúde Pública. Cuando publicás un artículo en Lancet o en otras revistas similares es porque conseguiste tener una idea original, conseguiste responder todas las cuestiones planteadas, etc., etc., y eso es un estímulo para que la gente siempre evolucione.

Por otro lado, creo que no se debería exigir que los posgrados publiquen solo en revistas A1. Al contrario, creo que es medio extraño que solo publiquen en esas revistas, normalmente no sucede eso. Lo más usual es que publiquen allí solo algunos artículos.

No queremos competir con las revistas extranjeras de primera línea, ni con las que están casi en la primera línea, sino que queremos competir con las que tienen un factor de impacto de 2 o 3 y que en general demoran mucho para publicar los artículos. Y esa es otra cosa que intentamos hacer, nuestra meta es reducir a seis meses el promedio de tiempo de publicación. Actualmente tenemos un promedio de un año, tal vez nueve meses, pero no tenemos ninguna esperanza de reducir el promedio a menos de seis meses. Entonces, ¿cómo es que vamos a competir con esas revistas que están con un factor de impacto un poco mayor que el nuestro? Ofreciendo fast tracking o, mejor dicho, la manera que encontramos de hacerlo. En las reuniones periódicas que realizamos con todos los editores asociados los orientamos para que estén atentos a las investigaciones que se están realizando en su área, para acercarse a esas personas que están presentando su investigación en congresos o conferencias y ofrecer la publicación en la revista del siguiente modo: "estamos muy interesados en publicar tu investigación y zqué es lo que te ofrecemos? En cuanto presenten el artículo, me voy a encargar del seguimiento en forma personal, me voy a comunicar diariamente con los revisores para conseguir una respuesta rápida y probablemente consigamos publicar el artículo en unos tres meses. $\mathrm{Si}$ lo mandan a otra revista, por lo menos va a tardar un año en publicarse". Y hemos conseguido algunas cosas, aunque ya sabemos que según el tipo de artículo que estén haciendo va para una revista o para otra. Por ejemplo, un estudio de cohorte en Pelotas, que encontró una relación entre amamantamiento y coeficiente intelectual, no lo van a mandar a la Revista de Saúde Pública, lo van a publicar en Lancet, ni queremos competir con eso.

Otro tema es que nuestro factor de impacto varía porque se producen fluctuaciones. El período de citación de nuestros artículos es largo. Las personas tardan en publicar, después tardan en citar, los proyectos de investigación se demoran también. Si querés citar un artículo, no podés porque todavía no lo terminaron. $Y$ nosotros pagamos ese precio porque el numerador de ese indicador, en nuestro caso se conforma con un alto porcentaje de citas que provienen de revistas brasileñas, y vienen con retraso. Además, si publicamos un suplemento aumenta mucho el denominador.

No vamos a pasar de un factor de impacto de 1 a 2 en uno o dos años. Eso va a llevar mucho tiempo porque el factor de impacto $-y$ esto es lo que le planteamos a la gente de SciELO- no se da en función de la visibilidad, sino de la calidad de la investigación que se haga en Brasil. Si la calidad de las investigaciones aumenta, la revista va a aumentar el índice de impacto, de lo contrario es un crecimiento artificial. El crecimiento real depende de la calidad de la investigación del artículo. Por ejemplo, en 
Brasil hay un estudio de cohorte que comenzó hace 30 años y ahora es uno de los estudios de cohorte más grandes de Brasil, entonces eso genera un tipo de producción que va a ser más citada. También sucede lo mismo si hay grandes centros o equipos de investigación interesados en cuestiones actuales. Por ejemplo, hicimos un número especial sobre producción científica en Brasil, y uno de los artículos realizó un análisis por áreas que identificó que ciertas áreas, como la entomología, tenían artículos muy citados. Al profundizar sobre el área de epidemiología nutricional mostró que se trataba de un grupo que comenzó a trabajar sobre obesidad con grupos del exterior, que había una persona que hizo una pasantía en San Pablo con nosotros y que se fue a Río de Janeiro y creó otro grupo allá sobre epidemiología nutricional. La gente de Pelotas también comenzó a trabajar más sobre epidemiología nutricional, y es eso lo que hace que el área tenga cierto crecimiento.

Lo que nosotros esperamos es que aumenten esas islas temáticas de excelencia. Por ejemplo, en el caso del sida no había gente investigando en Brasil y ahora hay mucha gente investigando, entonces ese grupo tiene condiciones para hacer trabajos que tengan impacto. Eso es lo que va a aumentar los índices de impacto de las revistas brasileñas, no se les puede reclamar a las revistas un desempeño disociado de la calidad de la investigación. Por eso, creo que hay que pensar en términos de "¿cómo mejoro la calidad de las investigaciones?" y no solo "¿cómo mejoro el impacto de las revistas?" Las revistas tienen que dar el máximo de sí mismas, tienen que hacer todo lo necesario, pero esos índices solo van a comenzar a aumentar, o van a tener un aumento sostenible, cuando las investigaciones tengan impacto.

VM: ¿Cuál es su opinión respecto de los nuevos requerimientos de internacionalización propuestos por SciELO Brasil?

CAM: A partir de la implementación de la idea de internacionalización, SciELO comenzó a proponer que las revistas tengan un porcentaje de editores asociados de instituciones no brasileñas. Nosotros vamos a lograrlo porque tenemos a muchos brasileños trabajando en el exterior e intentaremos resolverlo de ese modo. Ahora pensemos en un profesor de la Johns Hopkins University. Una parte del trabajo de cualquier profesor es ser editor científico o editor asociado de una revista, pero él tiene allá la American Journal of Clinical Nutrition, Journal of Nutrition, Public Health Nutrition, etc., y él quiere ser parte de esas revistas. ¿Por qué va a aceptar una invitación mía para ser editor asociado y trabajar para nuestra revista? Porque cuando él vaya a pelear su promoción -que en EE.UU. es un tema muy fuerte- y diga: "soy editor asociado de la Revista de Saúde Pública" -con el nombre de la revista así en portugués- le responderán: "Ah sí... y ¿qué pasó? ¿Las demás no te invitaron? [risas...] ¿Quién va a aceptar eso? Nadie va aceptar eso.

Ahora, en el área de física, por ejemplo, eso sí tiene sentido: si tengo una revista que publica artículos universales y estoy publicando artículos de Australia, entonces tiene sentido invitar a alguien de allá, porque incluso ni saben que la revista se edita en Brasil porque el nombre está en inglés, son revistas internacionales. Y eso está complicando las cosas, porque se quiere trasladar esa misma idea a otras áreas. Además están exigiendo otras cosas, como la profesionalización de los editores, que requieren de recursos adicionales que no disponemos. Si le pagáramos a un editor científico ¿cómo vamos a invitar a un editor asociado sin pagarle si va a hacer un trabajo similar? Entonces establecimos que todo el trabajo técnico-científico de los investigadores no es pago porque, si pagáramos eso, el costo sería absurdo.

La revista es de la Faculdade de Saúde Pública de la USP, y son las autoridades de la facultad quienes deben ratificar a los siete miembros del consejo editorial. En general, nosotros llevamos la propuesta y ellos la aprueban. El editor científico es elegido por esos siete miembros. Recientemente, Rita y yo fuimos reelectos y ya dijimos que era el último período, por lo que Leopoldo (José Leopoldo Ferreira Antunes) será quien nos suceda. Él 
tiene mucha más paciencia para discutir y dar cuenta de las exigencias de SciELO.

VM: ¿Cuál es el balance personal de la experiencia como editor de la Revista de Saúde Pública?

CAM: A mí me gusta mucho la investigación, que es lo que yo hago, y cuando asumí las tareas de la revista fue porque no había en ese momento otra persona... Cuando el profesor Forattini se enfermó, me dijo: “No hay caso, vas a tener que hacerte cargo, no hay otra persona". Y yo me resistía un poco [risas...] Pero hace ya casi 15 años que estoy como editor científico, los primeros cinco años estuve como viceeditor, mientras él continuaba siendo editor científico, luego él pasó a ser editor honorario y yo continué en su lugar.

VM: Bueno, ha sido un gran placer...

CAM: Les deseo mucha suerte a ustedes en su revista...

\section{FORMA DE CITAR}

Martinovich V. Práctica editorial contextualizada: Carlos Augusto Monteiro y la Revista de Saúde Pública. Salud Colectiva. 2016;12(2):295-304. DOI: 10.18294/sc.2016.980

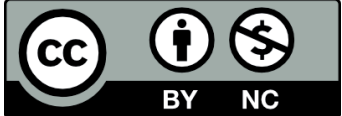

Este obra está bajo una licencia de Creative Commons Reconocimiento-NoComercial 4.0 Internacional. Reconocimiento - Permite copiar, distribuir y comunicar públicamente la obra. A cambio, se debe reconocer y citar al autor original. No Comercial - Esta obra no puede ser utilizada con finalidades comerciales, a menos que se obtenga el permiso. 